\title{
Design of a force-based controlled mobility on aerial vehicles for pest management
}

\author{
Laurent Reynaud ${ }^{\mathrm{a}, \mathrm{b}, *}$, Isabelle Guérin-Lassous ${ }^{\mathrm{b}}$ \\ ${ }^{a}$ Orange Labs, Lannion, France \\ ${ }^{b}$ Université de Lyon / LIP (ENS Lyon, CNRS, UCBL, INRIA), Lyon, France
}

\begin{abstract}
Vespa velutina, also known as the Asian hornet, is considered as an invasive species out of its native zone. In particular, since it preys on honey bees, its recent progression in Europe could soon pose a significant risk to the local apiculture activity. European beekeepers are therefore investigating adapted control strategies, including $V$. velutina nest destruction. Unfortunately, nest location pinpointing generally follows a manual process which can prove tedious, time-consuming and inaccurate. In this article, we propose the use of a network of micro aerial vehicles featuring autonomous and cooperative flight capabilities. We describe an adapted controlled mobility strategy and detail the design of our Virtual Force Protocol (VFP) which allows a swarm of vehicles to track and follow hornets to their nests, while maintaining connectivity through a wireless multi-hop communication route with a remote ground station used to store applicative data such as hornet trajectory and vehicle telemetry. In order to achieve the mission objectives with a minimum of vehicles, we identify through simulations appropriate value for the key parameters of VFP and discuss the obtained network performance.
\end{abstract}

Keywords: Controlled mobility, UAV networking, physics-based swarm intelligence

${ }^{*}$ Corresponding author

Email addresses: laurent.reynaud@orange.com (Laurent Reynaud), Isabelle.Guerin-Lassous@ens-lyon.fr (Isabelle Guérin-Lassous)

Preprint submitted to Journal of ${ }^{A} T_{E} X$ Templates

October 11, 2016 


\section{Introduction}

Vespa velutina, also known as the Asian or yellow-legged hornet, belongs to the insect order Hymenoptera, which also comprises wasps and bees. Although this hornet is native from Asia, it has recently extended its geographical range;

5 subspecies $V$. velutina ssp. nigritorax was for instance first observed in France in 2004 and is now widespread in about two thirds of the country [10. It is therefore considered as an invasive species and a major threat for honeybees, which it heavily preys upon [16]. Moreover, since this species has no natural predator outside its native zone, it can inflict all the more significant damage on domestic honeybee hives. As a result, stakeholders have actively been investigating different approaches for an efficient pest management strategy, with mixed results so far. In this regard, three promising research directions include selective trapping mechanisms, the use of narrow-spectrum parasitoids and most prominently, V. velutina nest elimination. However, this last method is largely complicated by the great variability of the nests, in terms of shape, size and location. Besides, nest destruction still essentially relies on a manual process: $V$. velutina specimens are usually captured around different domestic beehives, and when released, their trajectory and bearings are recorded and latter intersected, in order to estimate possible nest locations 29 .

In order to facilitate and speed up this technique, this article investigates the use of mobile robots and more specifically unmanned aerial vehicles (UAVs) to automatically track released hornets, and report in real time the related trajectories as well as other important data to a centralized host. In essence, UAVs have attracted a significant interest from both Academia and Industry and are often considered as a potential extension or substitution to operated networks. UAVs may greatly differ from their aerodynamic principles, size, autonomy and applicative features [17. Of these, the subcategory of micro UAVs propelled by rotors is increasingly used and tested in real-world scenarios where features such as cost efficiency, the existence of multiple degrees of freedom, so payload versatility and the ability to fly at low altitude, hover and keep a quasi- 
stationary position are seen as major assets. These capabilities have triggered the elaboration of numerous use cases, encompassing public protection and disaster relief [28, novel agriculture and forestry services (also referred as precision farming [18]), earth observation and environmental protection [7, aerial filming 35 and photography, temporary event services, unmanned delivery systems, etc.

In this study, we principally focus on the principles of controlled mobility 22, according to which embedded mobility schemes are empowered with the ability to steer UAVs equipped with a telecommunication payload and guide them where they can temporarily bring additional communication resource or increase network performance. Additionally, such mobile network nodes may also embed custom services, like precision farming and pest management applications, as discussed in this article. Further, we describe a mobility control protocol which we designed and named the Virtual Force Protocol (VFP). As the name suggests, VFP implements a system of virtual forces based on the relative 45 proximity between neighboring nodes. In this regard, we investigate through simulations how efficiently VFP can support a wireless multi-hop network of mobile nodes, including UAVs, in order to meet the requirements addressed by the considered pest management scenario.

This article is organized as follows: Section 2 surveys the state of the art related to autonomous mobility control strategies, while Section 3 presents the main applicative scenario that provides a realistic context to our study. At this point, the target network topology and equipment requirements to support this scenario are given, as well as a general view of the associated case flow. Section 4 delves deeper into the general principles that underlie the principles of mobility control algorithms based on virtual forces and gives an in-depth view of the design choices made for our VFP protocol which implements an efficient mobility control algorithm adapted to the considered applicative scenario. On this basis, Section 5 analyzes the VFP performance through representative network simulations. Finally, Section 6 concludes this study. 


\section{State of the Art}

This work is based on the underlying assumption that in order to realistically meet the considered pest management scenario requirements, mobile nodes need to feature advanced mobility mechanisms. To this end, we seek to use the ability of UAVs to easily maneuver on the designated area in order to fulfill two types of missions. First, UAVs feature a high number of degrees of freedom and therefore can avoid obstacles and efficiently track and pursue objects in motion, thanks to various types of range and vision sensors. On this matter, the recent progress on affordable and energy-efficient systems-on-a-chip (SoC) has been offering a growing on-board computational power even on small consumer multi-rotor UAVs 24, 28, which in turn has been giving an increased processing support for embedded and real-time computer vision [24, [25]. This in turn authorizes disruptive applicative perspectives, such as automatic refueling, soil sampling or retrieval of a payload in the context of delivery services 25, ball tracking systems for sporting events [26], the straightforward implementation of 75 a vision-based follow-me mode on leisure drones, along with any potential airborne service requiring target tracking. Secondly, UAVs can help bring wireless network connectivity where communication link disruptions are experienced, between two or more nodes in need of data transmission [17, 27. This later ability directly relates to the concept of controlled mobility, thereby giving a fresh perspective on network node mobility, which was until then considered more as a nuisance than an opportunity. With controlled mobility, nodes move where they can prove useful in terms of additional network resource [22].

Overall, many autonomous approaches based on that class of mobility schemes have been actively investigated in the last decades. Of these, a series of works proposed distributed path planning strategies featuring explicit network-oriented objectives. As such, a decentralized control scheme based on the estimation of a communication objective function gradient is presented in [30, with the goal to form communication chains and maximize network throughput by moving the relays on the basis of the perceived Signal-to-Noise Ratio (SNR). Likewise, the 
authors of [31] examine with graph-theoretic techniques a network connectivity problem to achieve a desired topology.

In this study, we focused on another distributed form of controlled mobility where node cooperation, which is not inherently based on network connectivity as an explicit requirement, may however result in valuable emergent behaviors [23. Among those, two prominent directions have been gaining momentum, respectively based on the concepts of stigmergic collaboration and physicomimetics: Stigmergy, which was first theorized by P. Grass in the late 1950s [9] during his research on termites, conventionally refers to the ability, for a swarm of vehicles, to indirectly coordinate and adopt an emergent behavior, via traces left in the environment. This is notably the case of pheromonebased mechanisms, which rely on the dissemination of specific messages, the pheromones [2, 8]. These messages are inherently associated to the specific location of their emission and commonly exhibit two key properties: signal additivity (two pheromones associated to the same location add up into a single signal with a stronger intensity) and decay (the intensity of a pheromone decreases with time). Applied to the domain of the controlled mobility of a swarm of vehicles, pheromones can encode any type of raw data such as vehicle or obstacle location, but also more synthetic information (e.g. path bottlenecks [2], the best trails leading to an applicative target [8], etc.). This type of scheme is therefore adapted for swarm vehicles such as mobile robots and UAVs, which can take advantage of pheromones found in their local environment to plan their individual trajectory accordingly. However, the practical applications of stigmergy to swarm vehicle path planning are significantly impaired by the difficulty to simply implement the interactions with the environment, which often ends up in either designing a centralized entity to manage those interactions or letting the individual network nodes exchange their partial view of the environment, at the expense of view inconsistencies and increased traffic overhead. In contrast, physics-based swarm intelligence, sometimes referred to as physicomimetics [1], is a class of controlled mobility mechanisms which primarily relies on the local interactions between neighboring nodes of the swarm. Although those interac- 
tions may be defined differently according to specific solutions, they share key commonalities:

- In addition to traditional forces (e.g. gravity, thrust and drag) they are subjected to, nodes seek to regularly evaluate the resulting virtual forces exerted by their neighbors in order to modify their respective acceleration, speed and location accordingly. In this regard, many definitions and designs were proposed for the concept of virtual forces in the literature, such as analogies with gravitational or electromagnetic forces [1, gas expansion models [3], liquid surface tension models [4, bio-inspired schemes [5] or custom distance-based forces [6], [20].

- To allow virtual force computation at each node, local information observation is performed via either close-range sensing or information exchange with surrounding nodes.

It is worth mentioning that unlike most proposed physicomimetics-based solutions, which assume large-scale swarms of nodes, and are typically adapted to the formation of lattices or grids of nodes, we propose in this paper a solution which is noticeably adapted to the formation of communication chains, in the context of a limited number of swarm elements which must be used as efficiently as possible.

\section{Scenario and main case flow}

In this section, we present the studied scenario and the different entities from the network architecture, along with their associated mobility patterns.

\subsection{Overview of the network topology}

In our scenario, locating $V$. velutina nests is made possible thanks to the prior capture of $V$. velutina specimens, around the bee hives which we seek to eventually protect by identifying and removing the considered nests. Captured hornet specimens are equipped with a visual marker (e.g. a lightweight 


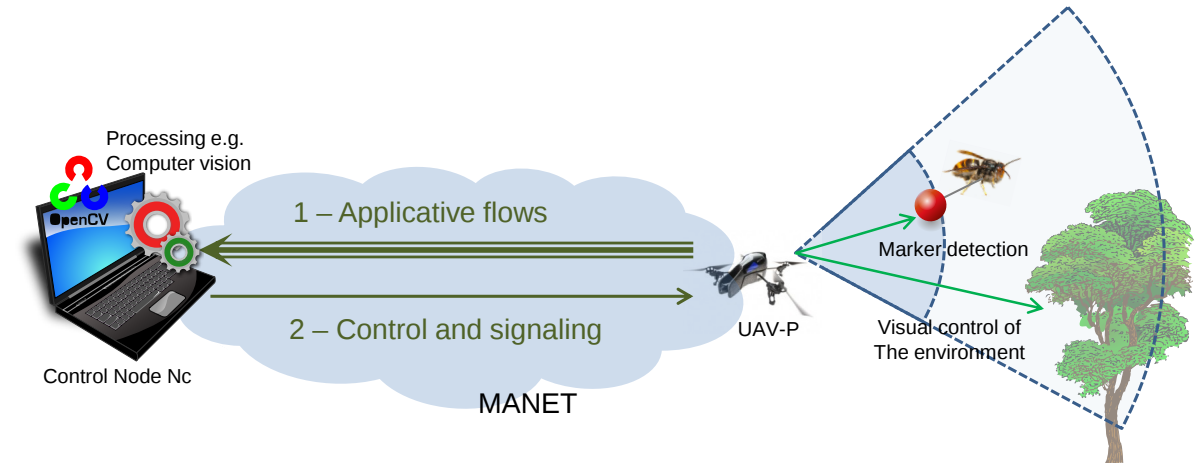

Figure 1: Tracking principles of a hornet by UAV-P, and communication flows between UAV-P and Nc.

polystyren bead or a small ribbon) which can be automatically tracked from the release point (ordinarily the zone of capture) to the destination point (which should hopefully be the nesting point). It should be noted that consistently with previous studies investigating manual nest identification based on trajectory intersections [29, hornets are here always assumed to follow a straight line between both endpoints. Besides, to support the proposed use case, we consider a network architecture encompassing the following set of nodes:

- A control node, Nc, which receives and displays all logging, tracing and telemetry flows from the other nodes. It is also meant to allow a human pilot to manually take control of any UAV of the swarm if required.

- A pursuit UAV, UAV-P, which is equipped with sensors and computer vision capabilities that enable the detection and tracking of the aforementioned visual markers. UAV-P is therefore able to estimate the trajectories followed by released $V$. velutina specimens. This information is sent to Nc along with UAV-P's telemetry data and other applicative flows, as illustrated by Fig. 1. Moreover, it is assumed in the context of this applicative scenario that a single UAV-P is required.

- Relay UAVs, UAV-R, which allow establishing and maintaining multihop communication routes between UAV-P and Nc, especially when they 


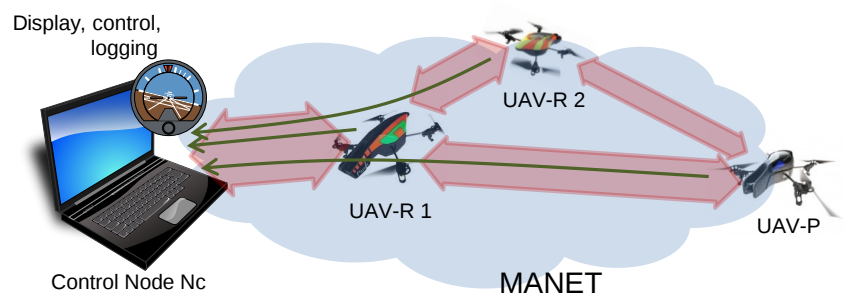

Figure 2: General communication flows between the active network nodes.

are not in direct radio transmission range. As shown by Fig. 2, this capability is supported by the combined use of a Mobile Ad Hoc Network (MANET) routing protocol and an adapted mobility control scheme.

- In addition, it is worth mentioning that supplementary nodes, named UAV-S, may act, on demand, as relay nodes (and in a broader perspective may fulfill any other applicative role). Those are parked in standby mode near Nc's location and able to immediately warm up and take off, when required.

Furthermore, all UAVs embed both an Inertial Measurement Unit (IMU) as well as an outside location system that enable the estimation of their position, speed and attitude (i.e. yaw, pitch and roll). Besides, network nodes (i.e. including Nc) are equipped with a standard wireless local area network (WLAN) communication interface (assumed to support IEEE 802.11 in this study).

\subsection{Mobility patterns}

Aside from node $\mathrm{Nc}$, which is assumed to remain still during the whole course of the scenario, three mobility schemes were designed for the UAVs. For UAV$\mathrm{P}$, the position estimation of the pursued hornet is directly used as an output measurement of the control loop feedback mechanism of the UAV's autopilot. In turn, the autopilot is able to perform the desired thrust corrections and to subsequently steer the aerial vehicle towards the desired coordinates, at a given distance of the estimated position of the tracked specimen. This mobility therefore solely depends on this estimated position when the considered target is 


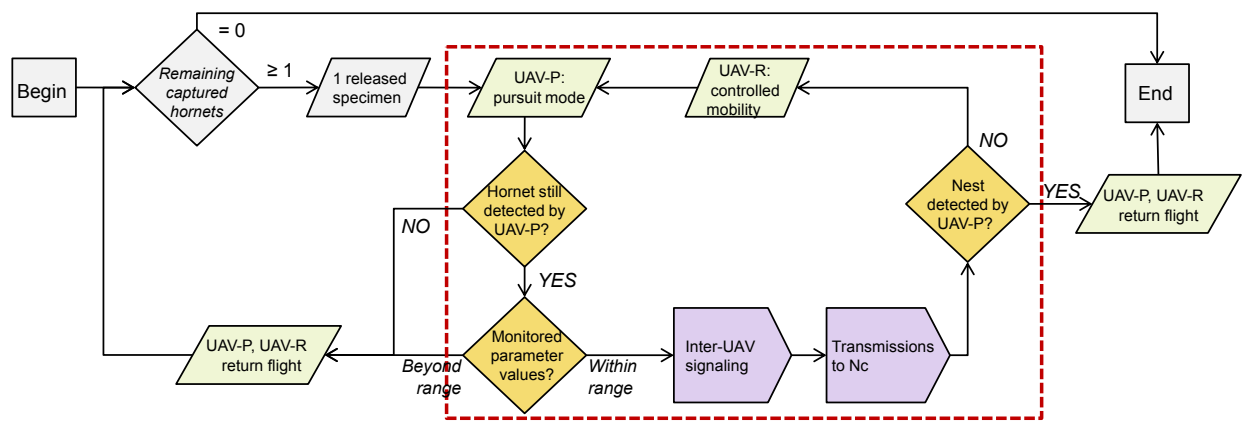

Figure 3: Representation of the main use case flow.

acquired by the computer vision procedures embedded on UAV-P. In addition, a series of exit cases are defined (e.g. loss of the target, detection of a stationary position for a threshold amount of time, depletion of the energy source, etc.) and will lead UAV-P to autonomously return to its starting position for maintenance, release of the next $V$. velutina specimen or for scenario termination, as illustrated by Fig. 3 .

Also, supplementary nodes UAV-S are initially located near the release site, and keep a static position until they are required and turn into relay nodes, UAV-R, as will be detailed in subsection 4.2. When no longer needed as relay nodes, those UAVs revert back to their UAV-S type, and autonomously return to their starting position.

Finally, relay nodes UAV-R feature an adapted mobility control scheme, which is the object of this study. As previously mentioned, this mobility strategy is intended to allow the formation of a reliable MANET, in particular between nodes UAV-P and Nc. In practice, relay nodes are steered where they are best needed to create the desired network topology.

\subsection{Investigated case flow}

Figure 3 gives the general chronology of the scenario case flow, through the representation of a sequence of high-level procedures. Those illustrate the detection and pursuit of a released hornet, and lead to either the detection of a nest or to the premature ending of the case flow. In the latter case, there 
between UAV-P and Nc. VFP is presented in details in the next section.

\section{Design of the Virtual Force Protocol}

\subsection{Virtual force principles}

Figure 4 first illustrates how we used the principles of virtual forces and physicomimetics to design a protocol able to lead to the desired mobility patterns for the UAV-R relay nodes. The upper part of the figure sketches how different force behaviors are defined around a node $\mathrm{N}$, corresponding to three attractive, friction and repulsive zones. First, any other node $\mathrm{O}$ located in either the attractive or repulsive zone is under the influence of N's virtual attraction/repulsion force $\vec{f}$, collinear with $\overrightarrow{O N}$ and which can be specified by a function representing the force intensity depending on the distance between both nodes. Although any force intensity profile may be used (in this regard, the dotted red curve illustrated in Fig. 4 is given as an example) but the piecewisedefined function $f:\left[0, d_{a}\right] \rightarrow\{-I, 0, I\}$ is considered for the context of this study:

$$
f(d)=\left\{\begin{array}{cc}
I & 0 \leq d \leq d_{r} \\
0 & d_{r}<d<d_{f} \\
-I & d_{f} \leq d \leq d_{a}
\end{array}\right.
$$

Secondly, a friction zone is defined where $f=0$, i.e. no attraction/repulsion is exerted by node $\mathrm{N}$ on its neighboring node $\mathrm{O}$. We however assume in this area 


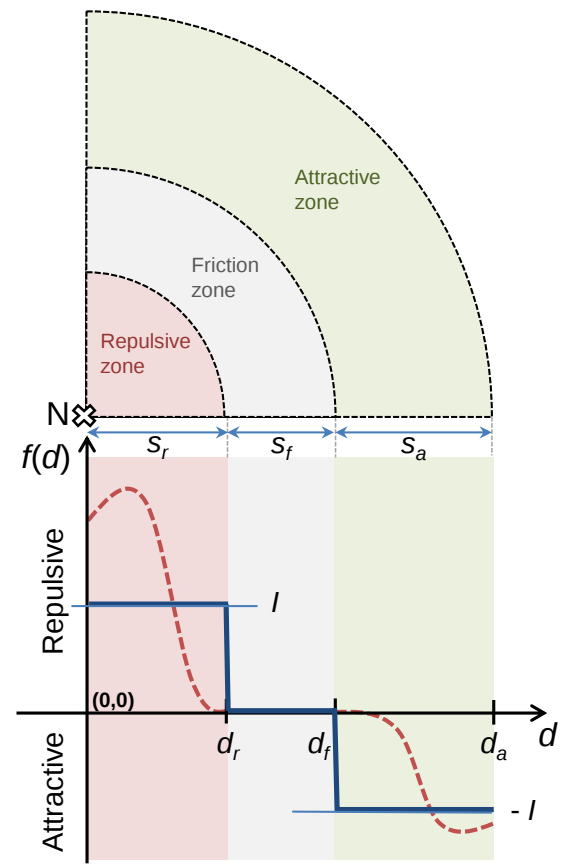

Figure 4: Definition of a virtual force exerted by a node $\mathrm{N}$ on its neighborhood.

the existence of a virtual friction force $\overrightarrow{f_{f r}}$ applied on node $\mathrm{O}$, collinear with O's velocity vector, and for which we define its intensity $f_{f r}=-C x$.

$$
f_{f r}=-C x \cdot f_{U}
$$

with $f_{U}=1 \mathrm{~N}$. In total, we adopt a simplified UAV mobility model, wherein a node can determine, based on the prior calculation of $\vec{f}$ and $\overrightarrow{f_{f r}}$ given by 11 and $(2)$, its next velocity vector $\vec{v}(t)$ :

$$
\vec{v}(t)=\vec{v}\left(t_{0}\right)+\left(t-t_{0}\right) \cdot \frac{\vec{f}+\overrightarrow{f_{f r}}}{m}
$$

With $m$, the node's mass and $\vec{v}\left(t_{0}\right)$, its velocity vector at previous time $t_{0}$.

\subsection{Protocol design}

Following the translation of the virtual force principles into a general purpose algorithm, we sought to design an efficient protocol solution, well-adapted 
Table 1: Description of the VFP beacon message

\begin{tabular}{|c|c|c|c|}
\hline NodeID & NodeType & ChainDst & AvailLinks \\
\hline PositionVector & \multicolumn{2}{|c|}{ VelocityVector } \\
\hline ChainPredecessor & \multicolumn{2}{|c|}{ ChainSuccessor } \\
\hline
\end{tabular}

to the considered applicative scenario [19]. In effect, we devised the Virtual Force Protocol (VFP) with the objective to support a distributed local information exchange through the use of specific beacon messages over the radio communication links. The regular emission of those control messages allow the formation and maintenance of a communication route from any (source, destination) pair that emits a communication request. Table 1 details the fields of a typical VFP beacon. In brief, the prominent fields give information about the unique application-level identifier of the emitting node (NodeID), its type (NodeType, which can be Nc, UAV-P, UAV-R or UAV-S), the identifier of the destination of the chain (ChainDst. If no valid identifier is given, the emitting node does not belong to a chain, at the time being), its position and velocity (PositionVector and VelocityVector).

Furthermore, in case the emitting node belongs to a chain, ChainPredecessor and ChainSuccessor give, if applicable, the unique identifiers of the previous and next nodes in the chain. If a node belongs to a chain and has no predecessor, it is the chain source. If it has no successor and the emitting node is not the destination, it may then need to request a new successor at a future time.

260 Finally, a non-zero value for the field AvailLinks is used to indicate that the emitting node is seeking to insert a new node into the chain.

We exemplify the use of these fields with the case illustrated by Fig. 5 which is compliant with the topology adopted for our applicative scenario. At an initial time $t_{1}$, all network nodes (Nc, UAV-P and a set of inactive UAVs of type UAV-S) are all located at a starting location, and node UAV-P flies away from this point. At this time, UAV-P belongs to a 2-node chain whose predecessor is Nc. After a while, UAV-P estimates, thanks to the VFP beacons 


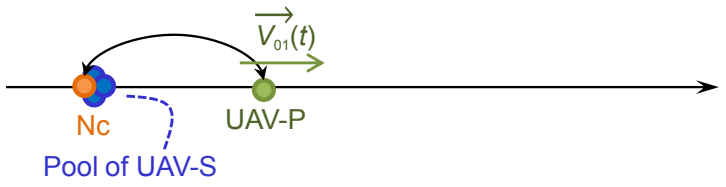

time $t_{1}$, chain $=(\mathrm{Nc}, \mathrm{UAV}-\mathrm{P})$

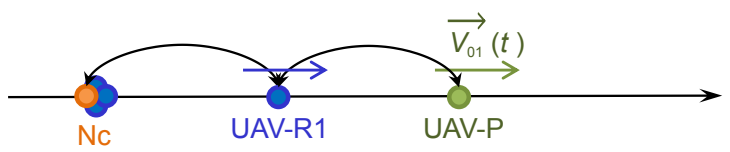

time $t_{2}$, chain $=(\mathrm{Nc}, \mathrm{UAV}-\mathrm{R} 1, \mathrm{UAV}-\mathrm{P})$

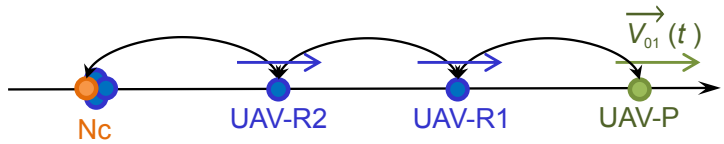

time $t_{3}$, chain $=(\mathrm{Nc}, \mathrm{UAV}-\mathrm{R} 2, \mathrm{UAV}-\mathrm{R} 1, \mathrm{UAV}-\mathrm{P})$

Figure 5: Formation of a multi-hop communication chain.

previously emitted by $\mathrm{Nc}$, that the distance with its successor is larger than a predetermined threshold $T h_{d \max }$. It then sets the field AvailLinks $=1$ in its own VFP beacons, meaning that UAV-P requests nearby UAV-S to advertise themselves, still via adapted VFP beacon values, as UAV-R candidates.

At time $t_{2}$, UAV-R1 is now elected by UAV-P, among the received candidatures, as a new intermediary node. The communication chain now consists of Nc, UAV-R1 and UAV-P and the ChainPredecessor and ChainSuccessor fields from the VFP beacons of each node is set accordingly.

Iteratively, this chain length can increase or decrease as required. For instance, Fig. 5 shows that at time $t_{3}$, another relay node, UAV-R2, was inserted in the chain. In effect, UAV-P continued to fly away from Nc, attracting UAV$\mathrm{R} 1$ and increasing the distance with Nc. As a result, UAV-R1 elected UAV-R2 as a new intermediary node (and its successor) in the considered chain.

Moreover, the VFP protocol also supports, in a similar fashion, the removal of a relay node from a communication chain. This repudiation process is here initiated by the predecessor of the considered relay node and is based on ade- 
quate values of the VFP beacon messages. Likewise, VFP features all necessary chain, e.g. when:

- A predecessor determines that the distance with its successor is smaller than a predetermined threshold $T h_{d m i n}$;

- A node from the chain detects a topology inconsistency with the information conveyed by a VFP beacon message from its predecessor (or successor) in the chain. In that case, in our implementation the chain is aggressively sanitized, i.e. a successor removes itself from the chain and reverts to the UAV-S state, while a predecessor will forget its successor and the rest of the chain, and initiate a new election process to designate a new successor.

\section{Performance evaluation}

In this section, we evaluate, by simulation, the performance of our proposed VFP solution. We first outline the simulation framework and related assumptions. Among those, we detail a set of four key parameters and their possible valuation range, thereby enabling a comprehensive characterization of how efficiently VFP-enabled network nodes interact to form the expected network topology. We then present the obtained simulation results, in the light of the impact of each key parameter. This performance study allows us to derive the best values to use for the key parameters.

\subsection{Simulation parameters}

305

In order to assess the performance of our force-based controlled mobility strategy, we carried out a simulation setup consistent with the applicative scenario described in Section 3 . On that account, we implemented the VFP protocol as well as the mobility schemes of UAV-S, UAV-P and UAV-R in the release 3.23 of the ns-3 network simulator. Table 2 gives a summary of the prominent parameter values. All 9 network nodes (Nc, UAV-P and 7 UAV-S nodes) are equipped with an IEEE $802.11 \mathrm{~b} / \mathrm{g}$ network interface and we assume lossless 
radio propagation for these communication channels. Moreover, all nodes are initially located at the center $\mathrm{C}(0,0,0)$ of the exploration area. During each simulation, Nc keeps a static position at point $\mathrm{C}$ and UAV-P follows a 1-dimension Random Waypoint (RWP) mobility pattern on the X axis within interval $I_{X}=$ [-350 m, $350 \mathrm{~m}]$ at constant speed, starting from C. It is worth noticing that this RWP mobility pattern allows modeling, for each simulation, a series of displacements along a straight line trajectory towards a random target position $\mathrm{T}$ of the hornet nest, assuming that:

- $\mathrm{T}$ is uniformly distributed in interval $I_{X}$;

- UAV-P position is here undifferentiated from that of the pursued hornet.

For the controlled mobility of the other nodes, VFP is configured so that each node exerts a virtual force with key parameters $d_{r}, d_{f}, d_{a}$, I and $C x$ valued as shown in Table 2. In particular, it can be noted that the boundary of the attractive area around a node, $d_{a}$, coincides with the radio range $r_{\text {range }}$ of its network interface. Moreover, we consider the threshold parameters $T h_{d \max }$ and $T h_{d m i n}$ which trigger the insertion or removal of an intermediate node in the multi-hop communication chain from UAV-P to Nc. To this end, it is worth mentioning that the multi-hop capability is supported by a MANET 330 routing protocol, and more specifically in this work, by the Optimized Link State Routing protocol (OLSR) [21]. We likewise set the force intensity $f(d)$ defined by (2) so that $I=1 \mathrm{~N}$. On a similar note, although the values of parameters $d_{f}, I, C x$ and $T h_{d m a x}$ are fixed in Table 2, in the rest of this section, we will also investigate in separate subsections how VFP performance varies with different values for each aforementioned parameter. To do so, we set up a Constant Bitrate (CBR) flow between nodes UAV-P and Nc, and perform through simulation a series of measurements on this flow. Notice that the choice of using a CBR flow is based on prior observations of actual data emitted by prototype quadcopters (notably in terms of control messages, telemetry and video flows), which remains relatively unwavering with time and therefore can be assumed constant in this study. Furthermore, we will consider two different 
Table 2: Main simulation parameters

\begin{tabular}{|l|l|l|}
\hline Parameter & Value(s) & Definition \\
\hline$c b$ & $\{10 \mathrm{kbit} / \mathrm{s}, 100 \mathrm{kbit} / \mathrm{s}\}$ & CBR bitrate \\
\hline$c p s$ & 100 bytes & CBR packet size \\
\hline$C x$ & 2 when fixed & friction coefficient \\
\hline$d_{a}$ & $100 \mathrm{~m}$ & attractive zone boundary \\
\hline$d_{f}$ & $\{50 \mathrm{~m}, 55 \mathrm{~m}, \cdots, 100 \mathrm{~m}\}$ & friction zone boundary \\
\hline$d_{r}$ & $50 \mathrm{~m}$ & repulsive zone boundary \\
\hline$I$ & $1 \mathrm{~N}$ when fixed & intensity parameter \\
\hline$L \times l$ & $1000 \mathrm{~m} \times 1000 \mathrm{~m}$ & exploration area \\
\hline$r_{\text {range }}$ & $100 \mathrm{~m}$ & radio range \\
\hline$t$ & $900 \mathrm{~s}$ & simulation time \\
\hline$T h_{\text {dmin }}$ & $40 \mathrm{~m}$ when fixed & distance threshold for removal \\
\hline$T h_{\text {dmax }}$ & $75 \mathrm{~m}$ when fixed & distance threshold for insertion \\
\hline
\end{tabular}

CBR bitrate values in order to study the performance of the VFP protocol in the context of low- (e.g. control messages and telemetry) and higher-capacity flows (e.g. a standard-definition video stream).

Furthermore, in terms of performance metrics, we use the following criteria:

- Packet Delivery Ratio (PDR) is defined here with respect to the CBR flow emitted by node UAV-P and sent to Nc. Our measured PDR is defined by the number of CBR packets received by destination Nc over the number of packets sent by source UAV-P.

- End-to-end delay: this delay is again related to the CBR flow between nodes UAV-P and Nc. This delay is calculated as the difference between the time of reception by Nc of the CBR packets at the application layer and the time of emission of these packets by UAV-P, still at the application layer.

- Stability of the VFP communication chains: the number of intermediate 
node insertion / removal events is measured, and allows assessing how stable the considered chain is. This criterion subsequently gives structuring information about the ability for the VFP mobility control strategy to efficiently meet the network topology objectives. the friction and attractive distances, through the variation of the single parameter $d_{f}$, which defines how large the friction and attraction zones are, via the 
following relations given by Fig. 4

$$
\begin{aligned}
& d_{f}=s_{r}+s_{f} \\
& d_{f}+s_{a}=d_{a}
\end{aligned}
$$

Parameters $s_{r}$ and $d_{a}$ being fixed (we conveniently set $d_{a}=r_{\text {range }}$ ), we therefore have a simple relation between the sizes of the friction and attraction zones, since:

$$
s_{a}=r_{\text {range }}-d_{f}
$$

Hence, studying the variations of $d_{f}$ in the range $\left[s_{r}, r_{\text {range }}\right]$ allows investigating different repartitions of friction and attractive zones, including the extreme cases of the absence of a friction zone (when $d_{f}=s_{r}$ ) or of an attractive zone (when $d_{f}=r_{\text {range }}$ ). The following couple of subsections present the performance results obtained for CBR bitrates of $10 \mathrm{kbit} / \mathrm{s}$ and $100 \mathrm{kbit} / \mathrm{s}$, in order to assess the ability of the VFP scheme to respectively support flows of different nature and bitrates in the context of the given scenario.

\subsubsection{Support of low-bitrate flows}

Figure 6 illustrates the simulation results regarding PDR and end-to-end delay with $d_{f}$, obtained with a CBR flow between nodes UAV-P and Nc, notably at a bitrate $c b=10 \mathrm{kbit} / \mathrm{s}$.

PDR. It is first worth observing that this PDR curve attains a maximum for the considered $d_{f}$ interval, with a PDR greater than $80 \%$ for $d_{f}=75$ to 80 $\mathrm{m}$. It then decreases when $d_{f}$ decreases from $d_{f}=75 \mathrm{~m}$ to the lower end of the considered interval. At $d_{f}=50 \mathrm{~m}$, a PDR around $60 \%$ is reached. Two reasons explain this local minimum: first, according to (4), this minimum value of $d_{f}$ results in $s_{f}=0$. Consequently, nearby nodes cannot stabilize anymore in the friction zone of the node that exerts virtual forces, which is a source of packet loss and which will significantly decrease the considered PDR. Secondly, 

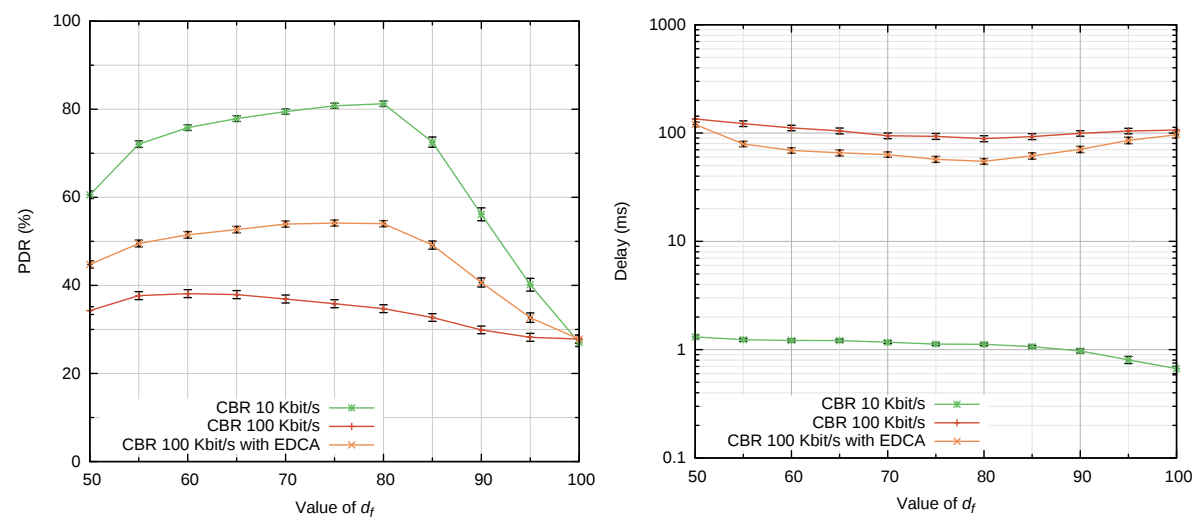

Figure 6: PDR (left) and end-to-end delay (right) of CBR packets received by node Nc, with 3 bitrates $(10 \mathrm{kbit} / \mathrm{s}, 100 \mathrm{kbit} / \mathrm{s}, 100 \mathrm{kbit} / \mathrm{s}$ with IEEE 802.11e EDCA [12] enabled)

according to (6), $s_{a}$ reaches here a maximum value and nodes will therefore attract nearby nodes closer to their own position. As a result, the distance between intermediate nodes of a multi-hop route will be smaller, and the number of hops for this route increases. Considering the assumptions of the simulation, and above all the use of a link-state routing protocol such as OLSR, increasing the average number of hops also increases the packet loss between source and destinations for this route. Likewise, from $d_{f}=80 \mathrm{~m}$ to the higher end of the considered interval, the PDR sharply declines, with a PDR bottoming at around $30 \%$. This absolute minimum can be easily explained, since according to (5), $d_{f}=100 \mathrm{~m}$ results in $s_{a}=0$, and in this extreme case, nodes cannot attract neighbors anymore. Therefore, with this value of $d_{f}$, the VFP protocol is not able to insert intermediate nodes and build communication chains of more than 1-hop between nodes UAV-P and Nc. Consequently, Nc receives UAV-P's CBR packets only when UAV-P is in direct communication range with Nc, which corresponds to the lowest value of the PDR, obtained for $d_{f}=100 \mathrm{~m}$.

End-to-end delay. Moreover, in complement with the aforementioned PDR, Fig. 6 (right) gives a representation of the end-to-end delay. Unsurprisingly, the delay is inversely proportional to the average number of hops (which, as 

slowly decreases as $d_{f}$ increases). The highest delay (around $1.3 \mathrm{~ms}$ ), is therefore obtained for $d_{f}=50 \mathrm{~m}$, and the lowest delay (around $0.5 \mathrm{~ms}$, i.e. about $40 \%$ of the highest delay) is reached for $d_{f}=100 \mathrm{~m}$. On that account, the assessment of the best range of value for the support of flows at $10 \mathrm{kbit} / \mathrm{s}$ (i.e. $d_{f}=75$ to $80 \mathrm{~m}$ ) is left unchanged by the analysis of the end-to-end delay of the CBR packets exchanged between nodes UAV-P and Nc.

Use of RTS/CTS. As a side remark, we also investigated the impact of the use of the Request to Send / Clear to Send (RTS/CTS) mechanism, whose objective, in the context of the IEEE 802.11 standard, is to mitigate the collision effects incurred by the hidden node problem [11. Simulation results with the use of RTS/CTS are identical regarding PDR. However, end-to-end delay (not represented for the sake of clarity) is here roughly doubled for all values of $d_{f}$. Although this delay, which is always less than $2.5 \mathrm{~ms}$, is still small and compatible with all kinds of applicative perspectives, it is worth stressing the counter-productive effect of the use of RTS/CTS in the context of our simulation scenario, which comforted us not to take into account the effects of this mechanism in the rest of the simulations.

\subsubsection{Support of flows with larger bitrates}

PDR and end-to-end delay. As previously mentioned in Section 3, the network may need to support communication flows with higher bitrates. To this end, we exemplify this capacity requirement by increasing the bitrate $c b$ of the CBR flow discussed in the previous subsection, from $10 \mathrm{kbit} / \mathrm{s}$ to $100 \mathrm{kbit} / \mathrm{s}$, leaving all other simulation parameters unchanged. Figure 6]also illustrates the results, both in terms of PDR and end-to-end delay, for that bitrate value. Clearly, in the considered $d_{f}$ interval, the PDR collapses between $30-40 \%$, while the endto-end delay sharply increases between 85-125 ms, significantly undermining the support of higher bitrates, low packet loss and delay insensitive communication flows. A more detailed analysis of the simulation traces reveals, in this case of CBR bitrates reaching $100 \mathrm{kbit} / \mathrm{s}$, a significant delay when broadcast packets 
need to be emitted while unicast frames are being retransmitted. Further, we verify that in this case, most retransmissions are caused by pairs of communicating nodes that mutually grow apart, because of their mobility patterns, and therefore become out of radio range. Consequently, unacknowledged frames are here likely to remain unacknowledged and will generally incur a maximum number of retransmissions (which is here set to 6 , consistently with the default ns-3 configuration) despite being not received by the destination nor improving the PDR metric previously discussed. Those unneeded retransmissions are detrimental to the queuing delays of broadcast packets (including beacon messages), which can amount, in our observations, for up to a few seconds. Because of these delays, nodes receive, at those times, outdated information from neighboring nodes, which can result in bogus node location with errors of up to 10-15 $\mathrm{m}$ and consequently undesirable network topologies, with a decreased PDR and increased end-to-end delay, as observed in Fig. 6.

Mitigation approaches. In this case of degraded performance, we therefore studied the behavior of the considered network under corrective actions, following two general directions:

- We first set the maximum Medium Access Control (MAC) frame retransmissions to zero, and verified that performance, both in terms of PDR and end-to-end delay, was brought back to that of the case of low CBR bitrate set at $c b=10 \mathrm{kbit} / \mathrm{s}$, with similar results.

- In a more realistic context, in which propagation channels are for instance not lossless, unicast frame retransmissions are customarily useful and must be enabled. In that regard, an alternative approach for the mitigation of their impact on broadcast packet queuing delays is to rely on prioritization mechanisms. As an illustration, we studied the use of the 4 access classes of IEEE 802.11e Enhanced Distributed Channel Access (EDCA) [12] and more precisely marked beacon broadcast packets with the high priority $A C_{-} V O$ access class, while the rest of the traffic remained associated with 
the default $A C_{-} B E$ best effort access class. As Fig. 6 shows, with beacon prioritization, PDR and end-to-end delay respectively reach about 55\% and $55-60 \mathrm{~ms}$ when $d_{f}$ is in the range 75 to $80 \mathrm{~m}$. Hence, the ability to transmit flows at $100 \mathrm{kbit} / \mathrm{s}$ is steadily improved, although it cannot favorably compare with the performance obtained in the case for which frame retransmissions are disabled.

490 Bearing those results in mind, it is also worth highlighting that the obtained VFP performance also depends on external factors. To begin with, there is room for improvement regarding the choice of an efficient routing protocol able to quickly set up an updated route when disruptions or failures are often experienced on a communication link. Likewise, an adapted routing solution could further benefit from link notification mechanisms such as MAC layer feedbacks [13], 14], which we did not include in our simulations. Similarly, the size cps of the CBR packets we used, i.e. 100 bytes, may need to be increased in a real-world scenario to avoid unnecessary header overhead and cope with higher bitrates. This aspect could therefore represent another valuable yet external factor for optimization.

As a consequence, it can be anticipated that beyond the context of our simulation scenario, the efficient support of higher bitrates highly depends on the use of VFP-independent mechanisms such as the use of an adapted routing protocol supporting low latency MAC layer feedback, broadband communication links and packet prioritization schemes. For this reason, regarding the VFP performance analysis solely, we will continue focusing, in the rest of this paper, on the support of flows with a bitrate $c b=10 \mathrm{kbit} / \mathrm{s}$, noticing that the efficient support of higher bitrates can be sought via the aforementioned external mechanisms.

\subsection{Impact of the force intensity parameter I}

In the previous simulations, we arbitrarily set $I$, a key parameter related to the virtual force intensity $f$ as defined in (1), to a fixed value. We now seek to evaluate the impact of $I$ on the overall VFP performance. To do so, we again 


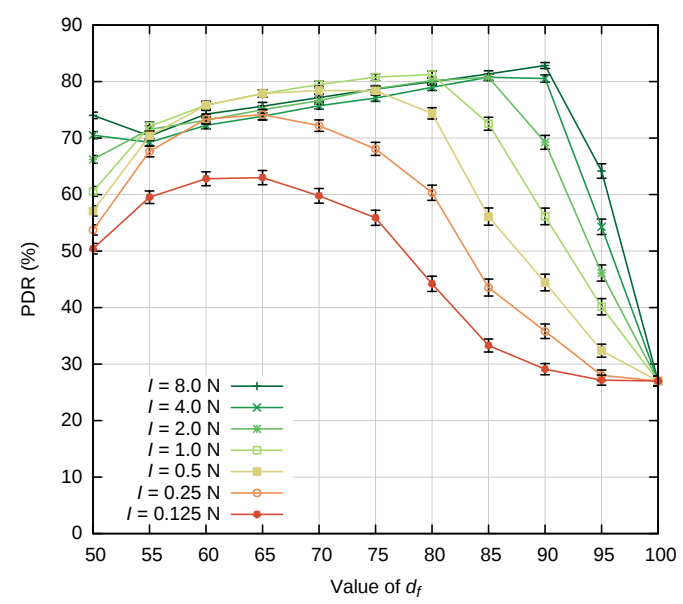

Figure 7: PDR of CBR packets sent by UAV-P and received by node Nc versus $d_{f}$, with different values of $I$.

investigated the PDR and end-to-end delay versus $d_{f}$ as previously analyzed, but also with a varying value of $I$, as illustrated by Fig. 7 where several PDR curves are represented with $I$ varying from $0.125 \mathrm{~N}$ to $8 \mathrm{~N}$. The case $I=1 \mathrm{~N}$, which was already analyzed in the previous simulations, is used as a comparative base.

$P D R$. These results first highlight that the optimal values for $d_{f}$ in terms of PDR varies with $I$. Moreover, it can be observed that those optimal $d_{f}$ values decrease when $I$ decreases from $1 \mathrm{~N}$ down $0.125 \mathrm{~N}$ (and below, although lower values are not represented in Fig. 7 for readability purposes). In this range of $I$, the best PDR results are therefore obtained with $I=1 \mathrm{~N}, d_{f}$ being in the range $[75 \mathrm{~m}, 80 \mathrm{~m}]$ as mentioned in the previous simulations. When $I$ is greater than $1 \mathrm{~N}$, the performance reaches a ceiling and the PDR curves remain almost unchanged for any value of $I$ above $8 \mathrm{~N}$. It is worth stressing that the range of acceptable values for $d_{f}$ shifts rightwards when $I$ increases. This could be perceived as an advantage since a higher value for $d_{f}$ will typically incur larger distances between neighboring intermediate nodes in a communication chain constructed with VFP. However, in practice, $I$ is limited in a real-world 
scenario by the accelerations and speeds achievable by the deployed UAVs [15].

End-to-end delay. The measured end-to-end delays for the same set of values for $I$ (not represented here) show that in all cases, CBR packets from UAV-P are received by $\mathrm{Nc}$ within a maximum $2 \mathrm{~ms}$ delay in average, for any value of $\left(d_{f}, I\right)$.

In consequence, this performance criterion does not challenge the observations previously made on the basis of the PDR metric. As a result, simulation results confirm that VFP performance depends on a judicious valuation for parameter I. More specifically, it can be observed that PDR performance increases when $I$ increases until the threshold $I=1 \mathrm{~N}$. A greater value for $I$ allows using

${ }_{540}$ larger values for $d_{f}$, enabling larger distances between intermediate nodes in a communication chain. However, $I$ cannot be indefinitely increased since this parameter is constrained by the inherent acceleration abilities of the mobile vehicles. For these reasons, we therefore choose for the rest of the study a conservative value of $I=1 \mathrm{~N}$, applicable to large set of UAV types of diverse propulsion systems.

\subsection{Impact of the friction coefficient $C x$}

In the same way that $I$ characterizes the attraction/repulsion zones around a VFP-enabled network node, dimensionless parameter $C x$ defines a virtual drag force $f_{f r}$ as specified in (2). We therefore sought to evaluate how network ${ }_{550}$ performance evolves with $C x$.

$P D R$. Figure 8 shows multiple PDR curves with $d_{f}$ in its habitual range and $C x$ varying from 0 to 32 . For this selected set of values, the best performance in terms of PDR is obtained for $C x=2$, when $d_{f}$ is in the range [75 m, 80 $\mathrm{m}]$, which further supports the use of this range of $d_{f}$ values as seen in the 555 previous simulations. Regarding the other $C x$ values, it is worth stressing that like physical friction forces, a greater $C x$ value allows a node to decelerate faster, and to subsequently stop its motion closer to the boundary of the friction area (i.e. closer to the repulsion/friction boundary of a repelling neighbor, or closer to friction/attraction of an attracting neighbor). Thus, with large $C x$ values, 
a node will tend to be close to one boundary and far from the other, incurring an imbalance in terms of intermediate node distance in a communication chain, depending on whether the neighboring node exerts a repulsive or an attractive virtual force. This behavior has a detrimental impact in our applicative scenario, for which attractions and repulsions are equally likely to occur. Fig. 8 confirms that the observed PDR sharply decreases when $C x$ increases from 2 to 32 and beyond (larger values of $C x$ are not represented but show almost identical results compared $C x=32$ ). Furthermore, it is worth noting that, similarly to what was stressed for parameter $I$, using a high value for $C x$ results into a strong virtual drag applied to the considered UAV, which physically requires the vehicle to apply a corresponding thrust, which, again, is inherently limited. Consequently, using high values of $C x$ is likely to become unrealistic if those values are kept inconsistent with the actual vehicle propulsive capabilities. In contrast, a lower value of $C x$ allows a node to enter deeper into (and even cross, if $C x$ is sufficiently small) the friction zone of a neighboring node. However, for smaller values of $575 C x$, this node will take more time to stabilize into the desired position, and will not even stabilize at all when $C x=0$. From $C x=2$ down to $C x=0$, simulation results corroborate this observation as PDR regularly declines for any given value of $d_{f}$.

End-to-end delay. As already noted for parameter $I$, simulations (not represented here) show that CBR packets are received within a maximum $1.5 \mathrm{~ms}$ end-to-end delay for any considered value of $\left(d_{f}, C x\right)$. Therefore, this performance criterion does not challenge the observations previously made on the basis of the PDR metric. To conclude, the determination of an appropriate value for parameter $C x$ is eased by the existence of a maximum, which corresponds, in the context of our scenario assumptions, manipulated value sets and simple force model, to $C x=2$.

\subsection{Impact of the distance thresholds}

We then finally sought to investigate the valuation of a last pair of key parameters for the VFP protocol, with the study of $T h_{d m i n}$ and $T h_{d m a x}$. Both 


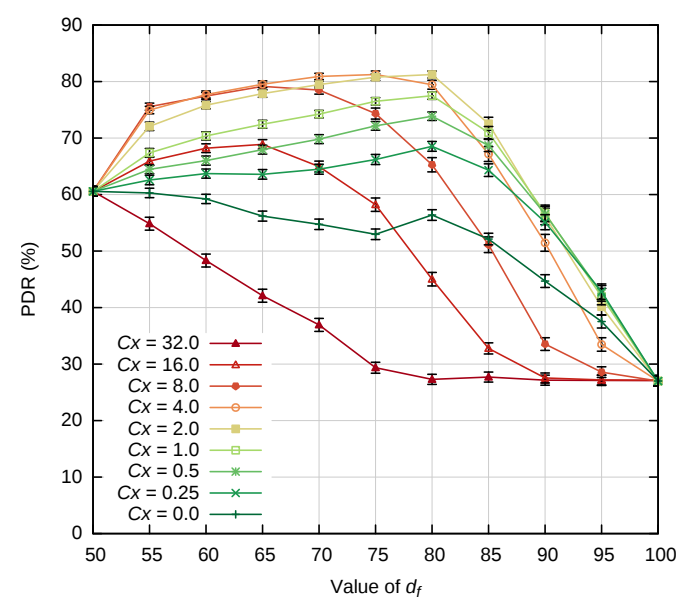

Figure 8: PDR of CBR packets sent by UAV-P and received by node Nc versus $d_{f}$, with different values of $C x$.

parameters represent distance thresholds which are respectively used by a VFPenabled node to determine whether its existing successor should be removed from a communication chain, or on the contrary if a new intermediate node should be inserted in that chain. Moreover, regarding the variation of $T h_{d \max }$ in the range $\left[d_{r}, d_{a}\right]$, the following behavior can be expected:

- A small value for $T h_{d m a x}$ close to $d_{r}$ incurs communication chains with small neighbor-to-neighbor link distances. For our applicative scenario, early UAV-R insertion may prove counter-productive since those newly inserted intermediate nodes may not be yet used as relays.

- In contrast, in the context of a large value for $T h_{d \max }$, a new UAV-R is inserted when link distance with its successor is already close to $d_{a}$, and therefore to $r_{\text {range }}$ in our scenario assumptions shown by Table 2 . Consequently, neighbor-to-neighbor link is already susceptible to be disrupted as soon as a new intermediate node is inserted, which is likely to significantly impair network performance.

605

We therefore carried out a series of simulations assuming $d_{f}=75 \mathrm{~m}\left(d_{f}\right.$ belonging to the optimal range previously determined in Subsection 5.1) and stud- 

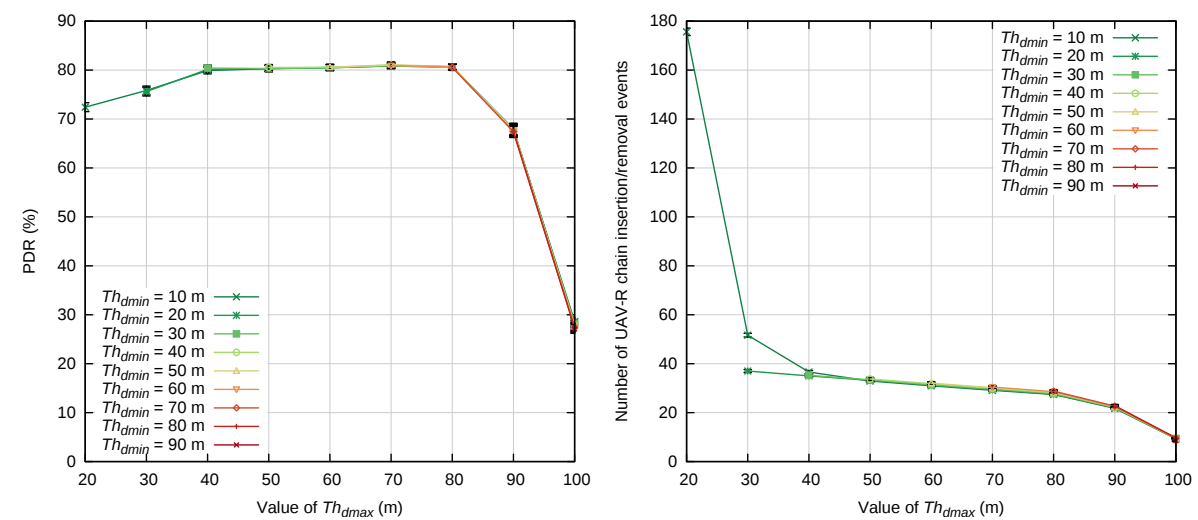

Figure 9: (left) PDR of packets received by Nc, (right) Insertion / removal events in a chain ied the variations of $T h_{d m i n}$ and $T h_{d \max }$ both in the interval $\left[d_{r}=50 \mathrm{~m}, d_{a}=\right.$ $100 \mathrm{~m}]$. It is important to note that the related simulations illustrated by Fig. 9 (left), 9 (right) and 10 only represent meaningful measures for which $T h_{d \min }<T h_{d \max }$.

PDR. Fig. 9 (left) depicts multiple $T h_{d m i n}$-dependent curves of PDR variations versus $T h_{d \max }$. Obviously, setting values for both thresholds so that $T h_{d \max }$ belongs in the range [ $40 \mathrm{~m}, 80 \mathrm{~m}$ ] leads to stable PDR performance. However, choosing smaller or larger values incurs a significant decrease in PDR results. Incidentally, it is worth noting that varying the value of $T h_{d m i n}$ has no significant impact on the obtained performance as long as $T h_{d \max }$ is in the appropriate value interval.

Stability. In addition, we investigated the stability of the communication chains formed via the support of VFP. More precisely, Fig. 9 (right) shows the number of UAV-R insertion / removal events into the chain for the selected values of pairs $\left(T h_{d \min }, T h_{d \max }\right)$. It should be mentioned that in the previously determined range $[40 \mathrm{~m}, 80 \mathrm{~m}]$ of stable PDR performance for $T h_{d m a x}$, the number of chain events regularly decreases when $T h_{d \max }$ increases: compared to the 34 insertion / removal events obtained for $T h_{d \max }=40 \mathrm{~m}$, this value is about ${ }_{625} 11 \%$ smaller for $T h_{d \max }=60 \mathrm{~m}$ and $21 \%$ less for $T h_{d \max }=80 \mathrm{~m}$. Since we 
previously observed with Fig. 9 (left) a stable PDR in the considered range, setting a value for $T h_{d \max }$ closer to the higher end of the considered interval, i.e. $T h_{d \max }=80 \mathrm{~m}$, is therefore preferable. As a side remark, two pairs of values for $T h_{d m i n}$ and $T h_{d \max }(10 \mathrm{~m}, 20 \mathrm{~m})$ and $(10 \mathrm{~m}, 30 \mathrm{~m})$ result into a steep increase of the observed chain events. Although both thresholds are then outside the recommended intervals, this increase requires a concise explanation: a detailed analysis of the simulation traces highlights that for these values, intermediate nodes can be subject to untimely attractions / repulsions in directions opposite to that of their predecessors, incurring undesirable chain events which explain the observed peaks for both pairs of values.

Number of unused UAVs. We finally focused on the number of required intermediate nodes to form a communication chain in the context of the applicative scenario. This last analysis also represents an important aspect of the study since VFP is designed to cope with a limited number of network nodes which must be used as efficiently as possible. To do so, Fig. 10 illustrates, for each considered value of pairs $\left(T h_{d \min }, T h_{d \max }\right)$, the average size of the set of unused relay nodes (in other words, the average number of UAV-S nodes). For the preferred range $[40 \mathrm{~m}, 80 \mathrm{~m}]$ of stable PDR performance, we can verify that the pool of UAV-S increases with $T h_{d \max }$ (this pool is about $7 \%$ larger for ${ }_{645} T h_{d \max }=80 \mathrm{~m}$ compared to its size for $\left.T h_{d \max }=40 \mathrm{~m}\right)$. Moreover, the set of unused UAVs also increases with $T h_{d \min }$ : for instance, at $T h_{d \max }=80 \mathrm{~m}$, setting $T h_{d \min }=70 \mathrm{~m}$ results into a pool $6 \%$ larger compared to its size for $T h_{d \min }=10 \mathrm{~m}$. Both results are naturally in line with our expectations, since a larger $T h_{d \max }$ incurs the late use of relay nodes while a larger $T h_{d m i n}$ results into an early release of unneeded intermediate nodes. Therefore, increasing those thresholds has a positive impact on the set of UAV-S nodes. In contrast, choosing lower values for the threshold pair leads to a steep decrease of the set of UAVs nodes (and, as previously seen with Fig. 9 (left) and Fig. 9 (right), is counter-productive since the PDR performance decreases while the number of UAV-R insertion / removal events largely increases). Lastly, using values above 


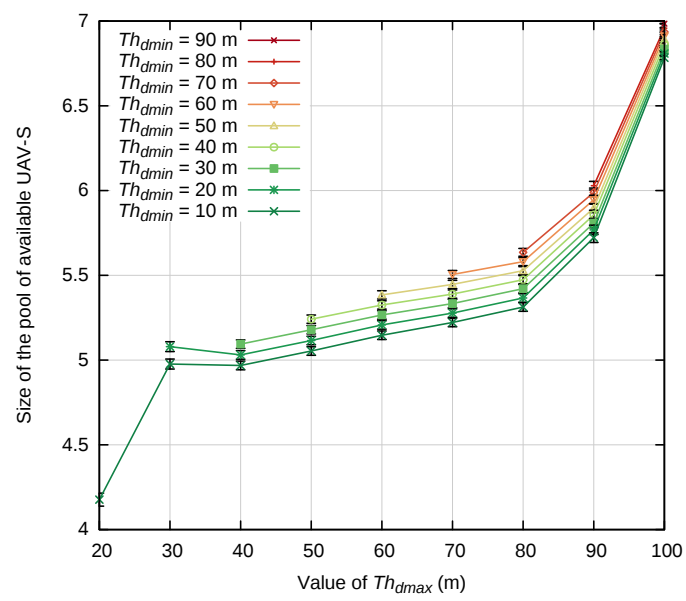

Figure 10: Number of unused UAV-S

the recommended interval for the pair of thresholds will dramatically increase the size of available UAV-S, at the expense of an equally dramatic decrease of the PDR performance.

As a result, simulation results also confirm that an appropriate valuation of the pair $\left(T h_{d \min }, T h_{d \max }\right)$ leads to an increase of VFP performance. While in strict terms of PDR results, $T h_{d \max }$ can belong to a relatively large interval $[40 \mathrm{~m}, 80 \mathrm{~m}]$ with the simple additional constraint $T h_{d m i n}<T h_{d m a x}$, the considerations of the stability of the formed chains as well as the size of unused UAV nodes allow advising for a value of $T h_{d \max }$ at the higher end of the aforementioned interval, with $T h_{d m i n}$ only slightly smaller. In the context of our simulations, the pair $\left(T h_{d \min }=70 \mathrm{~m}, T h_{d \max }=80 \mathrm{~m}\right)$ therefore represents an acceptable value. However, in real-world scenarios, the error inherent to all location systems must be taken into account in order to avoid that a small difference between both thresholds generates intermediate nodes insertion / removal 670 instabilities. Yet, in our simple and error-free model and with a minimum difference of $10 \mathrm{~m}$ between both thresholds, no effect of that nature was detected in our simulations. 


\section{Conclusion}

In this article, we described the VFP protocol, which allows a network of

675

685

690

mobile nodes such as a swarm of UAVs to efficiently form wireless multi-hop communication routes, thanks to a system of virtual forces based on the relative proximity between neighboring nodes. We more specifically investigated the context of an applicative scenario which aims at providing an automated determination of the nest locations of Vespa velutina, the Asian hornet, through

68 the tracking of released specimens, and the formation of communication chains between the tracking node and a ground station, designed to receive all communication flows. Moreover, we introduced in this work a series of key parameters for VFP, representing intrinsic properties of virtual forces such as force intensity, coefficient of friction, and multiple distance thresholds. We studied the impact of these parameter values on the overall protocol performance, in terms of packet delivery ratio, end-to-end delay, communication chain stability and ability to use as few relay nodes as possible. Through simulation analysis, we determined that in order to meet an acceptable VFP performance, optimal value intervals, consistent with UAV propulsive capabilities, can be identified. We then exemplified this determination by selecting adequate parameter values consistent with the general assumptions made in the context of our applicative scenario. Finally, we are now designing a quad-rotor system that features a system-on-a-chip able to execute a VFP implementation, which we are currently developing for this target architecture. In the future, we plan to further evaluate the performance of VFP on the basis of this experimental setup.

\section{References}

[1] W. M. Spears, D. F. Spears, J. C. Hamann, R. Heil, Distributed, PhysicsBased Control of Swarms of Vehicles, Auton. Robots, 17 (2/3) (2004) 137162. doi: 10.1023/B:AURO.0000033970.96785.f2 
[2] D. Payton, M. Daily, R. Estowski, M. Howard, C. Lee, Pheromone Robotics, Auton. Robots, 11 (3) (2001) 319324. doi: 10.1023/A:1012411712038

[3] J. Cheng, W. Cheng, R. Nagpal, Robust and self-repairing formation control for swarms of mobile agents, in: Proc. 20th national conf. on Artificial intell. (AAAI '05), Pittsburgh, USA (2005) 59-64.

[4] G. Elkaim, R. Kelbley, A Lightweight Formation Control Methodology for a Swarm of Non-Holonomic Vehicles, in: Proc. 2006 IEEE Aerospace Conference, Big Sky, USA (2006). doi: 10.1109/AERO.2006.1655803

[5] Y. Hanada, G. Lee, N. Chong, Adaptive Flocking of a Swarm of Robots Based on Local Interactions, in: Proc. 2007 IEEE Swarm Intelligence Symposium (SIS '07), Honolulu, USA (2007) 340-347. doi: 10.1109/SIS.2007.367957

[6] E. Yanmaz, Connectivity versus Area Coverage in Unmanned Aerial Vehicle Networks, in: Proc. 2012 IEEE International Conference on Communications (ICC 12), Ottawa, Canada (2012) 719723. doi: 10.1109/ICC.2012.6364585

[7] G. Varela, P. Caamamo, F. Orjales, A. Deibe, F. Lopez-Pena, R. J. Duro, Swarm intelligence based approach for real time UAV team coordination in search operations, in: Proc. Third World Congress on Nature and Biologically Inspired Computing, Salamanca, Spain (2011) 365370. doi: 10.1109/NaBIC.2011.6089619

[8] E. Pignaton de Freitas, T. Heimfarth, C. E. Pereira, A. Morado Ferreira, F. Rech Wagner, T. Larsson, Evaluation of coordination strategies for heterogeneous sensor networks aiming at surveillance applications, in Proc. 2009 IEEE Sensors, Christchurch, New Zealand (2009) 591596. doi: 10.1109/ICSENS.2009.5398314 
[9] P. Grassé, La reconstruction du nid et les coordinations interindividuelles; la théorie de la stigmergie, Insectes Sociaux, vol. 6 (1) (1959) 41-80. doi: 10.1007/BF02223791

730 [10] Distribution map of Vespa velutina in France, INPN Website, http:// inpn.mnhn.fr/espece/cd_nom/433589, 2016 (accessed 15.06.16).

[11] K.Xu, M. Gerla, S. Bae, Effectiveness of RTS/CTS handshake in IEEE 802.11 based ad hoc networks, Ad Hoc Networks journal, vol. 1 (1) (2003) 107123. doi: 10.1016/S1570-8705(03)00015-5

[12] A. Banchs, A. Azcorra, C. García, R. Cuevas, Applications and Challenges of the 802.11e EDCA Mechanism: An Experimental Study, IEEE Network, vol 19 (4) (2005) 52-58. doi: 10.1109/MNET.2005.1470683

[13] H. H. Shin, S. Lee, B.-S. Kim, Performance Improvement Using Self-LinkBreakage Announcement in Wireless Ad-hoc Networks, in: Proc. IEEE 16th Int. Conf. on Computational Science and Engineering (CSE 13), Sydney, Australia (2013) 208-212. doi:10.1109/CSE.2013.41

[14] B. Zhang, M. N. Shirazi, Implementation of explicit wireless loss notification using MAC-layer information, in: Proc. IEEE Wireless Comm. and Networking (WCNC '03), New Orleans, USA (2003) 1339-1343 vol.2. doi: 10.1109/WCNC.2003.1200568

[15] S.-J. Kim, I.-H. Whang, Acceleration Constraints for Maneuvering Formation Flight Trajectories, IEEE transactions on Aerospace and Electronic Systems, vol.48 (2) (2012) 1052-1060. doi: 10.1109/TAES.2012.6178048

[16] T. Mollet, C. de la Torre, Vespa velutina - The Asian hornet, Bee Craft (2007) 11-14.

[17] L. Reynaud, T. Rasheed, Deployable aerial communication networks: challenges for futuristic applications, in: Proc. 9th ACM Symposium on Performance Evaluation of Wireless Ad hoc, Sensor, and Ubiquitous Networks (PE-WASUN), Paphos, Cyprus (2012) 9-16. doi: 10.1145/2387027.2387030 
[18] B. S. Faical et al., Fine-Tuning of UAV Control Rules for Spraying Pesticides on Crop Fields, in: Proc. IEEE 26th Int. Conf. on Tools with Artificial Intell. (ICTAI), Limassol, Cyprus (2014) 527-533. doi: 10.1109/ICTAI.2014.85

[19] L. Reynaud, I. Guérin-Lassous, J. O. Calvar, Mobilité contrôlée pour la poursuite de frelons, in: Proc. 17èmes Rencontres Francophones sur les Aspects Algorithmiques des Télécommunications (ALGOTEL '15), Beaune, France (2015)

[20] D. V. Le , H. Oh, S. Yoon, VirFID: A Virtual Force (VF)-based InterestDriven moving phenomenon monitoring scheme using multiple mobile sensor nodes, in: Elsevier Ad Hoc Networks, vol. 27 (2015) p. 112132. doi: 10.1016/j.adhoc.2014.12.002

[21] T. Clausen, P. Jacquet, RFC3626: Optimized Link State Routing Protocol (OLSR), Experimental, http://www.ietf .org/rfc/rfc3626.txt

[22] W. Zhao, M. Ammar, E. Zegura, Controlling the mobility of multiple data

[25] M. Laiacker, M. Schwarzbach , K. Kondak, Automatic Aerial Retrieval of a Mobile Robot Using Optical Target Tracking and Localization. in: Proc. 2015 IEEE Aerospace Conference, Big Sky, USA (2015). doi: 10.1109/AERO.2015.7118992 
[26] H. Yadav, S. Srivastava, P. Mukherjee, B. Lall, A real-time ball trajectory follower using Robot Operating System, in: 3rd Int. Conf. on Image Information Processing (ICIIP), Waknaghat, India (2015). doi: 10.1109/ICIIP.2015.7414826

[27] O. K. Sahingoz, Networking Models in Flying Ad-Hoc Networks (FANETs): Concepts and Challenges. in: Journal of Intelligent and Robotic Systems, vol. 74 (1) (2014) 513-527. doi: 10.1007/s10846-013-9959-7

[28] C. Cambra, S. Sendra, J. Lloret, L. Parra, Ad hoc Network for Emergency Rescue System based on Unmanned Aerial Vehicles, in: Network Protocols and Algorithms, vol. 7 (4) (2015) 72-89. doi: 10.5296/npa.v7i4.8816

[29] J. Blot, Localisation et destruction des nids de frelons asiatiques, conception du réseau de signalement, in: Bulletin Technique Apicole, 34 (4) (2008) 205-210.

[30] C. Dixon, E. W. Frew, Optimizing cascaded chains of unmanned aircraft acting as communication relays, in: IEEE Selected Areas in Communications, vol. 30 (5) (2012) 883-898. doi: 10.1109/JSAC.2012.120605

[31] M.M. Zavlanos, M. B. Egerstedt, G. J. Pappas, Graph-theoretic connec800 tivity control of mobile robot networks, in: Proc. IEEE vol. 99 (9) (2011) 1525-1540. doi: 10.1109/JPROC.2011.2157884 